\title{
Hardy Inequalities and Non-explosion Results for Semigroups
}

\author{
Krzysztof Bogdan $^{1}$ - Bartłomiej Dyda ${ }^{1} \cdot$ Panki Kim $^{2}$
}

Received: 30 April 2015 / Accepted: 7 September 2015 / Published online: 15 September 2015

(C) The Author(s) 2015. This article is published with open access at Springerlink.com

\begin{abstract}
We prove non-explosion results for Schrödinger perturbations of symmetric transition densities and Hardy inequalities for their quadratic forms by using explicit supermedian functions of their semigroups.
\end{abstract}

Keywords Optimal Hardy equality $\cdot$ Transition density $\cdot$ Schrödinger perturbation

Mathematics Subject Classification (2010) Primary: 31C25 · 35B25; Secondary: 31C05 - 35B44

\section{Introduction}

Hardy-type inequalities are important in harmonic analysis, potential theory, functional analysis, partial differential equations and probability. In PDEs they are used to obtain a priori estimates, existence and regularity results [26] and to study qualitative properties and asymptotic behaviour of solutions [30]. In functional and harmonic analysis they yield

Krzysztof Bogdan and Bartłomiej Dyda were partially supported by NCN grant 2012/07/B/ST1/03356.

Panki Kim is supported by the National Research Foundation of Korea(NRF) grant funded by the Korea government(MSIP) (No. NRF-2015R1A4A1041675).

Bartłomiej Dyda

bdyda@pwr.wroc.pl

Krzysztof Bogdan

bogdan@pwr.edu.pl

Panki Kim

pkim@snu.ac.kr

1 Department of Mathematics, Wrocław University of Technology, Wybrzeże Wyspiańskiego 27, 50-370 Wrocław, Poland

2 Department of Mathematics, Seoul National University, Building 27, 1 Gwanak-ro, Gwanak-gu Seoul 151-747, Republic of Korea 
embedding theorems and interpolation theorems, e.g. Gagliardo-Nirenberg interpolation inequalities [23]. The connection of Hardy-type inequalities to the theory of superharmonic functions in analytic and probabilistic potential theory was studied, e.g., in [1, 6, 13, 16]. A general rule stemming from the work of P. Fitzsimmons [16] may be summarized as follows: if $\mathcal{L}$ is the generator of a symmetric Dirichlet form $\mathcal{E}$ and $h$ is superharmonic, i.e. $h \geq 0$ and $\mathcal{L} h \leq 0$, then $\mathcal{E}(u, u) \geq \int u^{2}(-\mathcal{L} h / h)$. The present paper gives an analogous connection in the setting of symmetric transition densities. When these are integrated against increasing weights in time and arbitrary weights in space, we obtain suitable (supermedian) functions $h$. This is one advantage of our approach, as compared to [16] — we propose a direct method for obtaining supermedian functions $h$. The resulting analogues $q$ of the Fitzsimmons' ratio $-\mathcal{L} h / h$ yield explicit Hardy inequalities, which in many cases are optimal. The approach is very general and the resulting Hardy inequality is automatically valid on the whole of the ambient $L^{2}$ space.

We also prove non-explosion results for Schrödinger perturbations of the original transition densities by the ratio $q$, namely we verify that $h$ is supermedian, in particular integrable, with respect to the perturbation. This is rather sharp, and has no analogue in [16]. For instance we recover the famous non-explosion result of Baras and Goldstein for $\Delta+(d / 2-1)^{2}|x|^{-2}$, cf. [3] and [27].

The results are illustrated by applications to transition densities with certain scaling properties.

The structure of the paper is as follows. In Theorem 1 of Section 2 we prove the nonexplosion result for Schrödinger perturbations. In Theorem 2 of Section 3 we prove the Hardy inequality. In fact, under mild additional assumptions we have a Hardy equality with an explicit remainder term. Sections 4, 5 and 6 present applications. In Section 4 we recover the classical Hardy equalities for the quadratic forms of the Laplacian and fractional Laplacian. For completeness we also recover the best constants and the corresponding remainder terms, as given by Filippas and Tertikas [15], Frank, Lieb and Seiringer [17] and Frank and Seiringer [18]. In Section 5 we consider transition densities with weak global scaling in the setting of metric spaces. These include a class of transition densities on fractal sets (Theorem 10 and Corollary 11) and transition densities of many unimodal Lévy processes on $\mathbb{R}^{d}$ (Corollary 12). We prove Hardy inequalities for their quadratic forms. In Section 6 we focus on transition densities with weak local scaling on $\mathbb{R}^{d}$. The corresponding Hardy inequality is stated in Theorem 13.

The calculations in Sections 4, 5 and 6, which produce explicit weights in Hardy inequalities, also give non-explosion results for specific Schrödinger perturbations of the corresponding transition densities by means of Theorem 1. These are stated in Corollaries 6 and 8 and Remarks 2, 5 and 6.

Currently our methods in Section 3 are confined to the (bilinear) $L^{2}$ setting. We refer to $[18,29]$ for other frameworks. Regarding further development, it is of interest to find relevant applications with less space homogeneity and scaling than required in the examples presented below, and to transition densities corresponding to specific boundary conditions (cf. the case of the censored processes in [12]), extend the class of considered time weights, prove explosion results for "supercritical" Schrödinger perturbations, and understand more completely when equality holds in our Hardy inequalities.

Below we use ":=" to indicate definitions, e.g. $a \wedge b:=\min \{a, b\}$ and $a \vee b:=\max \{a, b\}$. For two nonnegative functions $f$ and $g$ we write $f \approx g$ if there is a positive number $c$, called constant, such that $c^{-1} g \leq f \leq c g$. Such comparisons are usually called sharp estimates. We write $c=c(a, b, \ldots, z)$ to claim that $c$ may be so chosen to depend only on $a, b, \ldots, z$. For every function $f$, let $f_{+}:=f \vee 0$. For any open subset $D$ of the $d$-dimensional Euclidean 
space $\mathbb{R}^{d}$, we denote by $C_{c}^{\infty}(D)$ the space of smooth functions with compact supports in $D$, and by $C_{c}(D)$ the space of continuous functions with compact supports in $D$. In statements and proofs, $c_{i}$ denote constants whose exact values are unimportant. These are given anew in each statement and each proof.

\section{Non-explosion for Schrödinger Perturbations}

Let $(X, \mathcal{M}, m(d x))$ be a $\sigma$-finite measure space. Let $\mathcal{B}_{(0, \infty)}$ be the Borel $\sigma$-field on the halfline $(0, \infty)$. Let $p:(0, \infty) \times X \times X \rightarrow[0, \infty]$ be $\mathcal{B}_{(0, \infty)} \times \mathcal{M} \times \mathcal{M}$-measurable and symmetric:

$$
p_{t}(x, y)=p_{t}(y, x), \quad x, y \in X, \quad t>0,
$$

and let $p$ satisfy the Chapman-Kolmogorov equations:

$$
\int_{X} p_{s}(x, y) p_{t}(y, z) m(d y)=p_{s+t}(x, z), \quad x, z \in X, s, t>0,
$$

and assume that for every $t>0, x \in X, p_{t}(x, y) m(d y)$ is ( $\sigma$-finite) integral kernel. Let $f: \mathbb{R} \rightarrow[0, \infty)$ be non-decreasing, and let $f=0$ on $(-\infty, 0]$. We have $f^{\prime} \geq 0$ a.e., and

$$
f(a)+\int_{a}^{b} f^{\prime}(s) d s \leq f(b), \quad-\infty<a \leq b<\infty .
$$

Further, let $\mu$ be a nonnegative $\sigma$-finite measure on $(X, \mathcal{M})$. We put

$$
\begin{aligned}
p_{s} \mu(x) & =\int_{X} p_{s}(x, y) \mu(d y), \\
h(x) & =\int_{0}^{\infty} f(s) p_{s} \mu(x) d s .
\end{aligned}
$$

We denote, as usual, $p_{t} h(x)=\int_{X} p_{t}(x, y) h(y) m(d y)$. By Fubini-Tonelli and ChapmanKolmogorov, for $t>0$ and $x \in X$ we have

$$
\begin{aligned}
p_{t} h(x) & =\int_{t}^{\infty} f(s-t) p_{s} \mu(x) d s \\
& \leq \int_{t}^{\infty} f(s) p_{s} \mu(x) d s \\
& \leq h(x) .
\end{aligned}
$$

In this sense, $h$ is supermedian.

We define $q: X \rightarrow[0, \infty]$ as follows: $q(x)=0$ if $h(x)=0$ or $\infty$, else

$$
q(x)=\frac{1}{h(x)} \int_{0}^{\infty} f^{\prime}(s) p_{s} \mu(x) d s .
$$

For all $x \in X$ we thus have

$$
q(x) h(x) \leq \int_{0}^{\infty} f^{\prime}(s) p_{s} \mu(x) d s .
$$

We define the Schrödinger perturbation of $p$ by $q$ [8]:

$$
\tilde{p}=\sum_{n=0}^{\infty} p^{(n)},
$$


where $p_{t}^{(0)}(x, y)=p_{t}(x, y)$, and

$$
p_{t}^{(n)}(x, y)=\int_{0}^{t} \int_{X} p_{s}(x, z) q(z) p_{t-s}^{(n-1)}(z, y) m(d z) d s, \quad n \geq 1 .
$$

It is well-known that $\tilde{p}$ is a transition density [8].

Theorem 1 We have $\int_{X} \tilde{p}_{t}(x, y) h(y) m(d y) \leq h(x)$.

Proof For $n=0,1, \ldots$ and $t>0, x \in X$, we consider

$$
p_{t}^{(n)} h(x):=\int_{X} p_{t}^{(n)}(x, y) h(y) m(d y),
$$

and we claim that

$$
\sum_{k=0}^{n} p_{t}^{(k)} h(x) \leq h(x)
$$

By Eq. 7 this holds for $n=0$. By Eq. 11, Fubini-Tonelli, induction and Eq. 8,

$$
\begin{aligned}
\sum_{k=1}^{n+1} p_{t}^{(k)} h(x) & =\int_{X} \int_{0}^{t} \int_{X} p_{s}(x, z) q(z) \sum_{k=0}^{n} p_{t-s}^{(k)}(z, y) h(y) m(d y) d s m(d z) \\
& \leq \int_{0}^{t} \int_{X} p_{s}(x, z) q(z) h(z) m(d z) d s \\
& \leq \int_{0}^{t} \int_{X} p_{s}(x, z) \int_{0}^{\infty} f^{\prime}(u) \int_{X} p_{u}(z, w) \mu(d w) d u m(d z) d s . \\
& =\int_{0}^{t} \int_{0}^{\infty} f^{\prime}(u) p_{s+u} \mu(x) d u d s,
\end{aligned}
$$

where in the last passage we used Eqs. 2 and 4. By Eq. 3,

$$
\begin{aligned}
\sum_{k=1}^{n+1} p_{t}^{(k)} h(x) & \leq \int_{0}^{\infty} \int_{0}^{u \wedge t} f^{\prime}(u-s) d s p_{u} \mu(x) d u \\
& \leq \int_{0}^{\infty}[f(u)-f(u-u \wedge t)] p_{u} \mu(x) d u \\
& =\int_{0}^{\infty}[f(u)-f(u-t)] p_{u} \mu(x) d u,
\end{aligned}
$$

because $f(s)=0$ if $s \leq 0$. By this and Eq. 6 we obtain

$$
\begin{aligned}
\sum_{k=0}^{n+1} p_{t}^{(k)} h(x) \leq & \int_{t}^{\infty} f(u-t) p_{u} \mu(x) d u \\
& +\int_{0}^{\infty}[f(u)-f(u-t)] p_{u} \mu(x) d u \\
= & \int_{0}^{\infty} f(u) p_{u} \mu(x) d u=h(x) .
\end{aligned}
$$

The claim (12) is proved. The theorem follows by letting $n \rightarrow \infty$.

Remark 1 Theorem 1 asserts that $h$ is supermedian for $\tilde{p}$. This is much more than Eq. 7, but Eq. 7 may also be useful in applications [22, Lemma 5.2]. 
We shall see in Section 4 that the above construction gives integral finiteness (nonexplosion) results for specific Schrödinger perturbations with rather singular $q$, cf. Corollaries 6 and 8. In the next section $q$ will serve as an admissible weight in a Hardy inequality.

\section{Hardy Inequality}

Throughout this section we let $p, f, \mu, h$ and $q$ be as defined in Section 2. Additionally we shall assume that $p$ is Markovian, namely $\int_{X} p_{t}(x, y) m(d y) \leq 1$ for all $x \in X$. In short, $p$ is a subprobability transition density. By Holmgren criterion [25, Theorem 3, p. 176], we then have $p_{t} u \in L^{2}(m)$ for each $u \in L^{2}(m)$, in fact $\int_{X}\left[p_{t} u(x)\right]^{2} m(d x) \leq \int_{X} u(x)^{2} m(d x)$. Here $L^{2}(m)$ is the collection of all the real-valued square-integrable $\mathcal{M}$-measurable functions on $X$. As usual, we identify $u, v \in L^{2}(m)$ if $u=v m$-a.e. on $X$. The space (of equivalence classes) $L^{2}(m)$ is equipped with the scalar product $\langle u, v\rangle=\int_{X} u(x) v(x) m(d x)$. Since the semigroup of operators $\left(p_{t}, t>0\right)$ is self-adjoint and weakly measurable, we have

$$
\left\langle p_{t} u, u\right\rangle=\int_{[0, \infty)} e^{-\lambda t} d\left\langle P_{\lambda} u, u\right\rangle,
$$

where $P_{\lambda}$ is the spectral decomposition of the operators, see [21, Section 22.3]. For $u \in$ $L^{2}(m)$ and $t>0$ we let

$$
\mathcal{E}^{(t)}(u, u)=\frac{1}{t}\left\langle u-p_{t} u, u\right\rangle .
$$

By the spectral decomposition, $t \mapsto \mathcal{E}^{(t)}(u, u)$ is nonnegative and nonincreasing [19, Lemma 1.3.4], which allows to define the quadratic form of $p$,

$$
\mathcal{E}(u, u)=\lim _{t \rightarrow 0} \mathcal{E}^{(t)}(u, u), \quad u \in L^{2}(m) .
$$

The domain of the form is defined by the condition $\mathcal{E}(u, u)<\infty$ [19].

The following is a Hardy-type inequality with a remainder.

Theorem 2 If $u \in L^{2}(m)$ and $u=0$ on $\{x \in X: h(x)=0$ or $\infty\}$, then

$$
\begin{aligned}
& \mathcal{E}(u, u) \geq \int_{X} u(x)^{2} q(x) m(d x) \\
& +\liminf _{t \rightarrow 0} \int_{X} \int_{X} \frac{p_{t}(x, y)}{2 t}\left(\frac{u(x)}{h(x)}-\frac{u(y)}{h(y)}\right)^{2} h(y) h(x) m(d y) m(d x) .
\end{aligned}
$$

If $f(t)=t_{+}^{\beta}$ with $\beta \geq 0$ in (5) or, more generally, if $f$ is absolutely continuous and there are $\delta>0$ and $c<\infty$ such that

$$
[f(s)-f(s-t)] / t \leq c f^{\prime}(s) \quad \text { for all } s>0 \text { and } 0<t<\delta,
$$

then for every $u \in L^{2}(m)$

$$
\begin{aligned}
& \mathcal{E}(u, u)=\int u(x)^{2} q(x) m(d x) \\
& +\lim _{t \rightarrow 0} \int_{X} \int_{X} \frac{p_{t}(x, y)}{2 t}\left(\frac{u(x)}{h(x)}-\frac{u(y)}{h(y)}\right)^{2} h(y) h(x) m(d y) m(d x),
\end{aligned}
$$


Proof Let $v=u / h$, with the convention that $v(x)=0$ if $h(x)=0$ or $\infty$. Let $t>0$. We note that $|v h| \leq|u|$, thus $v h \in L^{2}(m)$ and by Eq. 7, $v p_{t} h \in L^{2}(m)$. We then have

$$
\mathcal{E}^{(t)}(v h, v h)=\left\langle v \frac{h-p_{t} h}{t}, v h\right\rangle+\left\langle\frac{v p_{t} h-p_{t}(v h)}{t}, v h\right\rangle=: I_{t}+J_{t} .
$$

By the definition of $J_{t}$ and the symmetry (1) of $p_{t}$,

$$
\begin{aligned}
J_{t} & =\frac{1}{t} \int_{X} \int_{X} p_{t}(x, y)[v(x)-v(y)] h(y) m(d y) v(x) h(x) m(d x) \\
& =\int_{X} \int_{X} \frac{p_{t}(x, y)}{2 t}[v(x)-v(y)]^{2} h(x) h(y) m(d x) m(d y) \geq 0 .
\end{aligned}
$$

To deal with $I_{t}$, we let $x \in X$, assume that $h(x)<\infty$, and consider

$$
\begin{aligned}
\left(h-p_{t} h\right)(x) & =\int_{0}^{\infty} f(s) p_{s} \mu(x) d s-\int_{0}^{\infty} f(s) p_{s+t} \mu(x) d s \\
& =\int_{0}^{\infty}[f(s)-f(s-t)] p_{s} \mu(x) d s .
\end{aligned}
$$

Thus,

$$
I_{t}=\int_{X} v^{2}(x) h(x) \int_{0}^{\infty} \frac{1}{t}[f(s)-f(s-t)] p_{s} \mu(x) d s m(d x) .
$$

By Eq. 13 and Fatou's lemma,

$$
\begin{aligned}
& \mathcal{E}(v h, v h) \geq \int_{X} \int_{0}^{\infty} f^{\prime}(s) p_{s} \mu(x) d s v^{2}(x) h(x) m(d x) \\
& +\liminf _{t \rightarrow 0} \int_{X} \int_{X} \frac{p_{t}(x, y)}{2 t}[v(x)-v(y)]^{2} h(y) h(x) m(d y) m(d x) \\
& =\int_{X} v^{2}(x) h^{2}(x) q(x) m(d x) \\
& +\liminf _{t \rightarrow 0} \int_{X} \int_{X} \frac{p_{t}(x, y)}{2 t}[v(x)-v(y)]^{2} h(y) h(x) m(d y) m(d x) .
\end{aligned}
$$

Since $u=0$ on $\{x \in X: h(x)=0$ or $\infty\}$, we have $u=v h$, hence $\mathcal{E}^{(t)}(u, u)=$ $\mathcal{E}^{(t)}(v h, v h)$ for all $t>0$, and so $\mathcal{E}(u, u)=\mathcal{E}(v h, v h)$. From Eq. 18 we obtain Eq. 14.

If $f$ is absolutely continuous on $\mathbb{R}$, then Eq. 3 becomes equality, and we return to Eq. 17 to analyse $I_{t}$ and $J_{t}$ more carefully. If $\int_{X} u(x)^{2} q(x) m(d x)<\infty$, which is satisfied in particular when $\mathcal{E}(u, u)<\infty$, and if Eq. 15 holds, then we can apply Lebesgue dominated convergence theorem to $I_{t}$. In view of Eqs. 13 and 17, the limit of $J_{t}$ then also exists, and we obtain Eq. 16. If $\int_{X} u(x)^{2} q(x) m(d x)=\infty$, then Eq. 18 trivially becomes equality. Finally, Eq. 15 holds for $f(t)=t_{+}^{\beta}$ with $\beta \geq 0$.

Corollary 3 For every $u \in L^{2}(m)$ we have $\mathcal{E}(u, u) \geq \int_{X} u(x)^{2} q(x) m(d x)$.

We are interested in non-zero quotients $q$. This calls for lower bounds of the numerator and upper bounds of the denominator. The following consequence of Eq. 14 applies when sharp estimates of $p$ are known.

Corollary 4 Assume there are a $\mathcal{B}_{(0, \infty)} \times \mathcal{M} \times \mathcal{M}$-measurable function $\bar{p}$ and a constant $c \geq 1$ such that for every $(t, x, y) \in(0, \infty) \times X \times X$,

$$
c^{-1} p_{t}(x, y) \leq \bar{p}_{t}(x, y) \leq c p_{t}(x, y) \text {. }
$$


Let

$$
\bar{h}(x)=\int_{0}^{\infty} \int_{X} f(s) \bar{p}_{s}(x, y) \mu(d y) d s,
$$

and let $\bar{q}(x)=0$ if $\bar{h}(x)=0$ or $\infty$, else let

$$
\bar{q}(x)=\frac{1}{\bar{h}(x)} \int_{0}^{\infty} f^{\prime}(s) \bar{p}_{s} \mu(x) d s .
$$

Then $c^{-1} h \leq \bar{h} \leq c h, c^{-2} q \leq \bar{q} \leq c^{2} q$, and for $u \in L^{2}(m)$ such that $u=0$ on $X \cap\{\bar{h}=0$ or $\bar{\infty}\}$, we have

$$
\mathcal{E}(u, u) \geq c^{-2} \int u(x)^{2} q(x) m(d x) .
$$

In the remainder of the paper we discuss applications of the results in Sections 2 and 3 to transition densities with certain scaling properties.

\section{Applications to (Fractional) Laplacian}

Let $0<\alpha<2, d \in \mathbb{N}, \mathcal{A}_{d,-\alpha}=2^{\alpha} \Gamma((d+\alpha) / 2) \pi^{-d / 2} /|\Gamma(-\alpha / 2)|$ and $v(x, y)=$ $\mathcal{A}_{d,-\alpha}|y-x|^{-d-\alpha}$, where $x, y \in \mathbb{R}^{d}$. Let $m(d x)=d x$, the Lebesgue measure on $\mathbb{R}^{d}$. Throughout this section, $g$ is the Gaussian kernel

$$
g_{t}(x)=(4 \pi t)^{-d / 2} e^{-|x|^{2} /(4 t)}, \quad t>0, \quad x \in \mathbb{R}^{d} .
$$

For $u \in L^{2}\left(\mathbb{R}^{d}, d x\right)$ we define

$$
\mathcal{E}(u, u)=\frac{1}{2} \int_{\mathbb{R}^{d}} \int_{\mathbb{R}^{d}}[u(x)-u(y)]^{2} v(x, y) d y d x .
$$

The important case $\beta=(d-\alpha) /(2 \alpha)$ in the following Hardy equality for the Dirichlet form of the fractional Laplacian was given by Frank, Lieb and Seiringer in [17, Proposition 4.1] (see [4] for another proof; see also [20]). In fact, [17, formula (4.3)] also covers the case of $(d-\alpha) /(2 \alpha) \leq \beta \leq(d / \alpha)-1$ and smooth compactly supported functions $u$ in the following Proposition. Our proof is different from that of [17, Proposition 4.1] because we do not use the Fourier transform.

Proposition 5 If $0<\alpha<d \wedge 2,0 \leq \beta \leq(d / \alpha)-1$ and $u \in L^{2}\left(\mathbb{R}^{d}\right)$, then

$$
\mathcal{E}(u, u)=C \int_{\mathbb{R}^{d}} \frac{u(x)^{2}}{|x|^{\alpha}} d x+\int_{\mathbb{R}^{d}} \int_{\mathbb{R}^{d}}\left[\frac{u(x)}{h(x)}-\frac{u(y)}{h(y)}\right]^{2} h(x) h(y) v(x, y) d y d x,
$$

where $C=2^{\alpha} \Gamma\left(\frac{d}{2}-\frac{\alpha \beta}{2}\right) \Gamma\left(\frac{\alpha(\beta+1)}{2}\right) \Gamma\left(\frac{d}{2}-\frac{\alpha(\beta+1)}{2}\right)^{-1} \Gamma\left(\frac{\alpha \beta}{2}\right)^{-1}, h(x)=|x|^{\alpha(\beta+1)-d}$. We get a maximal $C=2^{\alpha} \Gamma\left(\frac{d+\alpha}{4}\right)^{2} \Gamma\left(\frac{d-\alpha}{4}\right)^{-2}$ if $\beta=(d-\alpha) /(2 \alpha)$.

Proof Equation 21 is the Dirichlet form of the convolution semigroup of functions defined by subordination, that is we let $p_{t}(x, y)=p_{t}(y-x)$, where

$$
p_{t}(x)=\int_{0}^{\infty} g_{s}(x) \eta_{t}(s) d s
$$


$g$ is the Gaussian kernel defined in Eq. 20 and $\eta_{t} \geq 0$ is the density function of the distribution of the $\alpha / 2$-stable subordinator at time $t$, see, e.g., [5] and [19]. Thus, $\eta_{t}(s)=0$ for $s \leq 0$, and

$$
\int_{0}^{\infty} e^{-u s} \eta_{t}(s) d s=e^{-t u^{\alpha / 2}}, \quad u \geq 0 .
$$

Let $-1<\beta<d / \alpha-1$. The Laplace transform of $s \mapsto \int_{0}^{\infty} t^{\beta} \eta_{t}(s) d t$ is

$$
\begin{aligned}
\int_{0}^{\infty} \int_{0}^{\infty} t^{\beta} \eta_{t}(s) d t e^{-u s} d s & =\int_{0}^{\infty} t^{\beta} \int_{0}^{\infty} \eta_{t}(s) e^{-u s} d s d t=\int_{0}^{\infty} t^{\beta} e^{-t u^{\alpha / 2}} d t \\
& =\Gamma(\beta+1) u^{-\frac{\alpha(\beta+1)}{2}} .
\end{aligned}
$$

Since $\int_{0}^{\infty} e^{-u s} s^{\gamma} d s=\Gamma(\gamma+1) u^{-(\gamma+1)}$,

$$
\int_{0}^{\infty} t^{\beta} \eta_{t}(s) d t=\frac{\Gamma(\beta+1)}{\Gamma\left(\frac{\alpha(\beta+1)}{2}\right)} s^{\frac{\alpha(\beta+1)}{2}-1} .
$$

We consider $-\infty<\delta<d / 2-1$ and calculate the following integral for the Gaussian kernel by substituting $s=|x|^{2} /(4 t)$,

$$
\begin{aligned}
\int_{0}^{\infty} g_{t}(x) t^{\delta} d t & =\int_{0}^{\infty}(4 \pi t)^{-d / 2} e^{-|x|^{2} /(4 t)} t^{\delta} d t \\
& =(4 \pi)^{-d / 2}\left(\frac{|x|^{2}}{4}\right)^{\delta-d / 2+1} \int_{0}^{\infty} s^{d / 2-\delta-2} e^{-s} d s \\
& =4^{-\delta-1} \pi^{-d / 2}|x|^{2 \delta-d+2} \Gamma(d / 2-\delta-1) .
\end{aligned}
$$

For $f(t):=t_{+}^{\beta}$ and $\sigma$-finite Borel measure $\mu \geq 0$ on $\mathbb{R}^{d}$ we have

$$
\begin{aligned}
h(x) & :=\int_{0}^{\infty} \int_{\mathbb{R}^{d}} f(t) p_{t}(x-y) \mu(d y) d t \\
& =\int_{0}^{\infty} \int_{\mathbb{R}^{d}} t^{\beta} \int_{0}^{\infty} g_{s}(x-y) \eta_{t}(s) d s \mu(d y) d t \\
& =\int_{\mathbb{R}^{d}} \int_{0}^{\infty} \int_{0}^{\infty} t^{\beta} \eta_{t}(s) d t g_{s}(x-y) d s \mu(d y) \\
& =\int_{\mathbb{R}^{d}} \int_{0}^{\infty} \frac{\Gamma(\beta+1)}{\Gamma\left(\frac{\alpha(\beta+1)}{2}\right)} s^{\frac{\alpha(\beta+1)}{2}-1} g_{s}(x-y) d s \mu(d y) \\
& =\frac{\Gamma(\beta+1)}{\Gamma\left(\frac{\alpha(\beta+1)}{2}\right)} \frac{\Gamma\left(\frac{d}{2}-\frac{\alpha(\beta+1)}{2}\right)}{4^{\frac{\alpha(\beta+1)}{2}} \pi^{d / 2}} \int_{\mathbb{R}^{d}}|x-y|^{\alpha(\beta+1)-d} \mu(d y),
\end{aligned}
$$

where in the last two equalities we assume $\alpha(\beta+1) / 2-1<d / 2-1$ and use Eqs. 23 and 24 . If, furthermore, $\beta \geq 0$, then by the same calculation

$$
\begin{aligned}
\int_{0}^{\infty} \int_{\mathbb{R}^{d}} & f^{\prime}(t) p_{t}(x, y) \mu(d y) d t \\
& =\beta \frac{\Gamma(\beta)}{\Gamma\left(\frac{\alpha \beta}{2}\right)} 4^{-\frac{\alpha \beta}{2}} \pi^{-d / 2} \Gamma\left(\frac{d}{2}-\frac{\alpha \beta}{2}\right) \int_{\mathbb{R}^{d}}|x-y|^{\alpha \beta-d} \mu(d y) .
\end{aligned}
$$


Here the expression is zero if $\beta=0$. If $\mu=\delta_{0}$, then we get

$$
h(x)=\frac{\Gamma(\beta+1)}{\Gamma\left(\frac{\alpha(\beta+1)}{2}\right)} \frac{\Gamma\left(\frac{d}{2}-\frac{\alpha(\beta+1)}{2}\right)}{4^{\frac{\alpha(\beta+1)}{2}} \pi^{d / 2}}|x|^{\alpha(\beta+1)-d}
$$

and

$$
\begin{aligned}
q(x) & =\frac{1}{h(x)} \int_{0}^{\infty} \int_{\mathbb{R}^{d}} f^{\prime}(t) p_{t}(x, y) \mu(d y) d t \\
& =\frac{2^{\alpha} \Gamma\left(\frac{d}{2}-\frac{\alpha \beta}{2}\right) \Gamma\left(\frac{\alpha(\beta+1)}{2}\right)}{\Gamma\left(\frac{d}{2}-\frac{\alpha(\beta+1)}{2}\right) \Gamma\left(\frac{\alpha \beta}{2}\right)}|x|^{-\alpha} .
\end{aligned}
$$

By homogeneity, we may assume $h(x)=|x|^{\alpha(\beta+1)-d}$, without changing $q$. By the second statement of Theorem 2, it remains to show that

$$
\begin{aligned}
\lim _{t \rightarrow 0} \int_{\mathbb{R}^{d}} \int_{\mathbb{R}^{d}} \frac{p_{t}(x, y)}{2 t}\left[\frac{u(x)}{h(x)}-\frac{u(y)}{h(y)}\right]^{2} h(y) h(x) d y d x \\
=\frac{1}{2} \int_{\mathbb{R}^{d}} \int_{\mathbb{R}^{d}}\left[\frac{u(x)}{h(x)}-\frac{u(y)}{h(y)}\right]^{2} h(y) h(x) v(x, y) d y d x .
\end{aligned}
$$

Since $p_{t}(x, y) / t \leq v(x, y)$ [7] and $p_{t}(x, y) / t \rightarrow v(x, y)$ as $t \rightarrow 0$, Eq. 27 follows either by the dominated convergence theorem, if the right hand side of Eq. 27 is finite, or - in the opposite case - by Fatou's lemma. If $\alpha \beta=(d-\alpha) / 2$, then we obtain $h(x)=|x|^{-(d-\alpha) / 2}$ and

$$
q(x)=\frac{2^{\alpha} \Gamma\left(\frac{d+\alpha}{4}\right)^{2}}{\Gamma\left(\frac{d-\alpha}{4}\right)^{2}}|x|^{-\alpha} .
$$

This is optimal in the sense that

$$
r(t):=\frac{\Gamma\left(\frac{d}{2}-t\right) \Gamma\left(t+\frac{\alpha}{2}\right)}{\Gamma\left(\frac{d}{2}-\frac{\alpha}{2}-t\right) \Gamma(t)}, \quad t \in\left(0, \frac{d-\alpha}{2}\right),
$$

is maximal for $t=\frac{d-\alpha}{4}$, cf. (26). Indeed, by [2, (1.2.13)],

$$
\frac{\Gamma^{\prime}(x)}{\Gamma(x)}=-\gamma-\sum_{k=0}^{\infty}\left(\frac{1}{x+k}-\frac{1}{k+1}\right),
$$

where $\gamma$ is the Euler-Mascheroni constant, and so the derivative of $\log r$ is

$$
\begin{aligned}
\frac{r^{\prime}(t)}{r(t)} & =-\sum_{k=0}^{\infty}\left(\frac{-1}{d / 2-t+k}+\frac{1}{t+\alpha / 2+k}+\frac{1}{d / 2-\alpha / 2-t+k}-\frac{1}{t+k}\right) \\
& =\sum_{k=0}^{\infty} \frac{\alpha(4 k+d)(d-\alpha-4 t)}{(t+k)(d-2 t+2 k)(d-\alpha-2 t+2 k)(2 t+2 k+\alpha)} .
\end{aligned}
$$

It follows that $r^{\prime}(t)>0$ if $t<\frac{d-\alpha}{4}$ and $r^{\prime}(t)<0$ if $t>\frac{d-\alpha}{4}$, hence the maximum of $r$ is at $\frac{d-\alpha}{4}$. (A different proof is given in [17, Lemma 3.2].)

Finally, the statement of the proposition is trivial for $\beta=d / \alpha-1$. 
Corollary 6 If $0 \leq r \leq d-\alpha, x \in \mathbb{R}^{d}$ and $t>0$, then

$$
\int_{\mathbb{R}^{d}} p_{t}(y-x)|y|^{-r} d y \leq|x|^{-r} .
$$

If $0<r<d-\alpha, x \in \mathbb{R}^{d}, t>0, \beta=(d-\alpha-r) / \alpha, q$ is given by $E q .26$ and $\tilde{p}$ is given by Eq. 10, then

$$
\int_{\mathbb{R}^{d}} \tilde{p}_{t}(y-x)|y|^{-r} d y \leq|x|^{-r} .
$$

Proof By Eq. 7 and the proof of Proposition 5, we get the first estimate. The second estimate is stronger, because $\tilde{p} \geq p$, cf. (10), and it follows from Theorem 1 , cf. the proof of Proposition 5. We do not formulate the second estimate for $r=0$ and $d-\alpha$, because the extension suggested by Eq. 26 reduces to a special case of the first estimate.

For completeness we now give Hardy equalities for the Dirichlet form of the Laplacian in $\mathbb{R}^{d}$. Namely, Eq. 29 below is the optimal classical Hardy equality with remainder, and Eq. 28 is its slight extension, in the spirit of Proposition 5. For the equality (29), see for example [15, formula (2.3)], [18, Section 2.3] or [4]. Equality (28) may also be considered as a corollary of $[18$, Section 2.3$]$.

Proposition 7 Suppose $d \geq 3$ and $0 \leq \gamma \leq d-2$. For $u \in W^{1,2}\left(\mathbb{R}^{d}\right)$,

$$
\int_{\mathbb{R}^{d}}|\nabla u(x)|^{2} d x=\gamma(d-2-\gamma) \int_{\mathbb{R}^{d}} \frac{u(x)^{2}}{|x|^{2}} d x+\int_{\mathbb{R}^{d}}\left|h(x) \nabla \frac{u}{h}(x)\right|^{2} d x,
$$

where $h(x)=|x|^{\gamma+2-d}$. In particular,

$$
\int_{\mathbb{R}^{d}}|\nabla u(x)|^{2} d x=\frac{(d-2)^{2}}{4} \int_{\mathbb{R}^{d}} \frac{u(x)^{2}}{|x|^{2}} d x+\left.\left.\int_{\mathbb{R}^{d}}|| x\right|^{\frac{2-d}{2}} \nabla \frac{u(x)}{|x|^{(2-d) / 2}}\right|^{2} d x .
$$

Proof The first inequality is trivial for $\gamma=d-2$, so let $0 \leq \gamma<d-2$. We first prove that for $u \in L^{2}\left(\mathbb{R}^{d}, d x\right)$,

$$
\begin{aligned}
\mathcal{C}(u, u)= & \gamma(d-2-\gamma) \int_{\mathbb{R}^{d}} \frac{u(x)^{2}}{|x|^{2}} d x \\
& +\lim _{t \rightarrow 0} \int_{\mathbb{R}^{d}} \int_{\mathbb{R}^{d}} \frac{g_{t}(x, y)}{2 t}\left(\frac{u}{h}(x)-\frac{u}{h}(y)\right)^{2} h(y) h(x) d y d x,
\end{aligned}
$$

where $g$ is the Gaussian kernel defined in Eq. 20, and $\mathcal{C}$ is the corresponding quadratic form. Even simpler than in the proof of Proposition 5, we let $f(t)=t^{\gamma / 2}$ and $\mu=\delta_{0}$, obtaining

$$
\begin{aligned}
h(x) & :=\int_{0}^{\infty} f(s) g_{s} \mu(x) d s=\int_{0}^{\infty} \int_{\mathbb{R}^{d}} f(s) g_{s}(x-y) \mu(d y) d s \\
& =\int_{\mathbb{R}^{d}} 4^{-\gamma / 2-1} \pi^{-d / 2}|x-y|^{\gamma-d+2} \Gamma(d / 2-\gamma / 2-1) \mu(d y) \\
& =4^{-\gamma / 2-1} \pi^{-d / 2}|x|^{\gamma-d+2} \Gamma(d / 2-\gamma / 2-1), \\
\int_{0}^{\infty} f^{\prime}(s) g_{s} \mu(x) d s & =\frac{\gamma}{2} 4^{-\gamma / 2}|x|^{\gamma-d} \pi^{-d / 2} \Gamma(d / 2-\gamma / 2), \\
q(x) & =\frac{\int_{0}^{\infty} f^{\prime}(s) g_{s} \mu(x) d s}{h(x)}=\frac{\gamma(d-2-\gamma)}{|x|^{2}} .
\end{aligned}
$$


By Theorem 2 we get Eq. 30. Since the quadratic form of the Gaussian semigroup is the classical Dirichlet integral, taking $\gamma=(d-2) / 2$ and $q(x)=(d-2)^{2} /\left(4|x|^{2}\right)$ we recover the classical Hardy inequality:

$$
\int_{\mathbb{R}^{d}}|\nabla u(x)|^{2} d x \geq \frac{(d-2)^{2}}{4} \int_{\mathbb{R}^{d}} \frac{u(x)^{2}}{|x|^{2}} d x, \quad u \in L^{2}\left(\mathbb{R}^{d}, d x\right) .
$$

We, however, desire Eq. 28. It is cumbersome to directly prove the convergence of Eq. 30 to Eq. $28^{1}$. Here is an approach based on calculus. For $u \in C_{c}^{\infty}\left(\mathbb{R}^{d} \backslash\{0\}\right)$ we have

$$
\begin{gathered}
\partial_{j}\left(|x|^{d-2-\gamma} u(x)\right)=(d-2-\gamma)|x|^{d-4-\gamma} u(x) x_{j}+|x|^{d-2-\gamma} u_{j}(x), \\
\left|\nabla\left(|x|^{d-2-\gamma} u(x)\right)\right|^{2}=|x|^{2(d-4-\gamma)}\left[(d-2-\gamma)^{2} u(x)^{2}|x|^{2}+|x|^{4}|\nabla u(x)|^{2}\right. \\
\left.+(d-2-\gamma)\left\langle\nabla\left(u^{2}\right)(x), x\right\rangle|x|^{2}\right],
\end{gathered}
$$

hence

$$
\begin{aligned}
\int_{\mathbb{R}^{d}}\left|\nabla \frac{u}{h}(x)\right|^{2} h(x)^{2} d x= & \int_{\mathbb{R}^{d}}\left|\nabla\left(|x|^{d-2-\gamma} u(x)\right)\right|^{2}|x|^{2(\gamma+2-d)} d x \\
= & (d-2-\gamma)^{2} \int_{\mathbb{R}^{d}} u(x)^{2}|x|^{-2} d x+\int_{\mathbb{R}^{d}}|\nabla u(x)|^{2} d x \\
& +(d-2-\gamma) \int_{\mathbb{R}^{d}}\left\langle\nabla\left(u^{2}\right)(x),|x|^{-2} x\right\rangle d x .
\end{aligned}
$$

Since $\operatorname{div}\left(|x|^{-2} x\right)=(d-2)|x|^{-2}$, the divergence theorem yields Eq. 28. We then extend Eq. 28 to $u \in C_{c}^{\infty}\left(\mathbb{R}^{d}\right)$ as follows. Let $\psi \in C_{c}^{\infty}\left(\mathbb{R}^{d}\right)$ be such that $0 \leq \psi \leq 1, \psi(x)=1$ if $|x| \leq 1, \psi(x)=0$ if $|x| \geq 2$. Let $u_{n}(x)=u(x)[1-\psi(n x)], n \in \mathbb{N}$. We note the local integrability of $|x|^{-2}$ in $\mathbb{R}^{d}$ with $d \geq 3$. We let $n \rightarrow \infty$ and have Eq. 28 hold for $u$ by using the convergence in $L^{2}\left(|x|^{-2} d x\right)$, inequality $\left|\nabla u_{n}(x)\right| \leq|\nabla u(x)|+c_{1}|u(x)||x|^{-1}$, the identity $h(x) \nabla\left(u_{n} / h\right)(x)=\nabla u_{n}(x)-u_{n}(x)[\nabla h(x)] / h(x)$ for $x \neq 0$, the observation that $|\nabla h(x)| / h(x) \leq c|x|^{-2}$ and the dominated convergence theorem. We can now extend Eq. 28 to $u \in W^{1,2}\left(\mathbb{R}^{d}\right)$. Indeed, assume that $C_{c}^{\infty}\left(\mathbb{R}^{d}\right) \ni v_{n} \rightarrow u$ and $\nabla v_{n} \rightarrow g$ in $L^{2}\left(\mathbb{R}^{d}\right)$ as $n \rightarrow \infty$, so that $g=\nabla u$ in the sense of distributions. We have that $h(x) \nabla\left(v_{n} / h\right)(x)=\nabla v_{n}(x)-v_{n}(x)[\nabla h(x)] / h(x) \rightarrow g-u[\nabla h(x)] / h(x)$ in $L^{2}\left(\mathbb{R}^{d}\right)$. The latter limit is $h \nabla(u / h)$, as we understand it. We obtain the desired extension of Eq. 28. As a byproduct we actually see the convergence of the last term in Eq. 30. Taking $\gamma=(d-2) / 2$ in Eq. 28 yields Eq. 29.

We note that Eq. 32 holds for all $u \in L^{2}\left(\mathbb{R}^{d}\right)$.

Corollary 8 If $0 \leq r \leq d-2, x \in \mathbb{R}^{d}$ and $t>0$, then

$$
\int_{\mathbb{R}^{d}} g_{t}(y-x)|y|^{-r} d y \leq|x|^{-r} \text {. }
$$

If $0<r<d-2, x \in \mathbb{R}^{d}, t>0, \beta=(d-2-r) / 2$, $q$ is given by Eq. 31, and $\tilde{g}$ is the Schrödinger perturbation of $g$ by $q$ as in $E q$. 10, then

$$
\int_{\mathbb{R}^{d}} \tilde{g}_{t}(y-x)|y|^{-r} d y \leq|x|^{-r} .
$$

The proof is similar to that of Corollary 6 and is left to the reader.

\footnotetext{
${ }^{1}$ But see a comment before $[4,(1.6)]$ and our conclusion below.
} 


\section{Applications to Transition Densities with Global Scaling}

In this section we show how sharp estimates of transition densities satisfying certain scaling conditions imply Hardy inequalities. In particular we give Hardy inequalities for symmetric jump processes on metric measure space studied in [11], and for unimodal Lévy processes recently estimated in [7]. In what follows we assume that $\phi:[0, \infty) \rightarrow[0, \infty)$ is nondecreasing and left-continuous, $\phi(0)=0, \phi(x)>0$ if $x>0$ and $\phi\left(\infty^{-}\right):=\lim _{x \rightarrow \infty} \phi(x)=$ $\infty$. We denote, as usual,

$$
\phi^{-1}(u)=\inf \{s>0: \phi(s)>u\}, \quad u \geq 0 .
$$

Here is a simple observation, which we give without proof.

Lemma 9 Let $r, t \geq 0$. We have $t \geq \phi(r)$ if and only if $\phi^{-1}(t) \geq r$.

We see that $\phi^{-1}$ is upper semicontinuous, hence right-continuous, $\phi^{-1}\left(\infty^{-}\right)=\infty$, $\phi\left(\phi^{-1}(u)\right) \leq u$ and $\phi^{-1}(\phi(s)) \geq s$ for $s, u \geq 0$. If $\phi$ is continuous, then $\phi\left(\phi^{-1}(u)\right)=u$, and if $\phi$ is strictly increasing, then $\phi^{-1}(\phi(s))=s$ for $s, u \geq 0$. Both these conditions typically hold in our applications, and then $\phi^{-1}$ is the genuine inverse function.

We first recall, after [7, Section 3], [24, Section 2] and [31, (2.7) and (2.20)], the following weak scaling conditions. We say that a function $\varphi:[0, \infty) \rightarrow[0, \infty)$ satisfies the global weak lower scaling condition if there are numbers $\underline{\alpha} \in \mathbb{R}$ and $\underline{c} \in(0,1]$, such that

$$
\varphi(\lambda \theta) \geq \underline{c} \lambda \underline{\alpha} \varphi(\theta), \quad \lambda \geq 1, \quad \theta>0 .
$$

We then write $\varphi \in \operatorname{WLSC}(\underline{\alpha}, \underline{c})$. Put differently, $\varphi(R) / \varphi(r) \geq \underline{c}(R / r)^{\underline{\alpha}}, 0<r \leq R$. The global weak upper scaling condition holds if there are numbers $\bar{\alpha} \in \mathbb{R}$ and $\bar{c} \in[1, \infty)$ such that

$$
\varphi(\lambda \theta) \leq \bar{c} \lambda^{\bar{\alpha}} \varphi(\theta), \quad \lambda \geq 1, \quad \theta>0,
$$

or $\varphi(R) / \varphi(r) \leq \bar{c}(R / r)^{\bar{\alpha}}, 0<r \leq R$. In short, $\varphi \in \operatorname{WUSC}(\bar{\alpha}, \bar{c})$. We note that $\varphi$ has the lower scaling if and only if $\varphi(\theta) / \theta^{\underline{\alpha}}$ is almost increasing, i.e. comparable with a nondecreasing function on $[0, \infty)$, and $\varphi$ has the upper scaling if and only if $\varphi(\theta) / \theta^{\bar{\alpha}}$ is almost decreasing, see [7, Lemma 11].

Let $(F, \rho, m)$ be a metric measure space with metric $\rho$ and Radon measure $m$ with full support. We denote $B(x, r)=\{y \in F: \rho(x, y)<r\}$ and assume that there is a nondecreasing function $V:[0, \infty) \rightarrow[0, \infty)$ such that $V(0)=0, V(r)>0$ for $r>0$, and

$$
c_{1} V(r) \leq m(B(x, r)) \leq c_{2} V(r) \text { for all } x \in F \text { and } r \geq 0 .
$$

We call $V$ the volume function.

Theorem 10 Let $p$ be a symmetric subprobability transition density on $F$, with Dirichlet form $\mathcal{E}$, and assume that

$$
p_{t}(x, y) \approx \frac{1}{V\left(\phi^{-1}(t)\right)} \wedge \frac{t}{V(\rho(x, y)) \phi(\rho(x, y))}, \quad t>0, \quad x, y \in F,
$$

where $\phi, V:[0, \infty) \rightarrow(0, \infty)$ are non-decreasing, positive on $(0, \infty), \phi(0)=V(0)=$ $0, \phi\left(\infty^{-}\right)=\infty$ and $V$ satisfies Eq. 35. If $\underline{A}>\bar{\alpha}>0, V \in \operatorname{WLSC}(\underline{A}, \underline{C})$ and $\phi \in$ WUSC $(\bar{\alpha}, \bar{c})$, then there is $C>0$ such that

$$
\mathcal{E}(u, u) \geq C \int_{F} \frac{u(x)^{2}}{\phi(\rho(x, y))} m(d x), \quad y \in F, \quad u \in L^{2}(F, m) .
$$


Proof Let $y \in F$ and $u \in L^{2}(F, m)$. The constants in the estimates below are independent of $y$ and $u$. Let $0<\beta<\underline{A} / \bar{\alpha}-1$ and define

$$
h(x)=\int_{0}^{\infty} t^{\beta} p_{t}(x, y) d t, \quad x \in F .
$$

We shall prove that

$$
\begin{aligned}
& \mathcal{E}(u, u) \approx \int_{F} \frac{u(x)^{2}}{\phi(\rho(x, y))} m(d x) \\
& +\liminf _{t \rightarrow 0} \int_{F} \int_{F} \frac{p_{t}(x, z)}{2 t}\left(\frac{u(x)}{h(x)}-\frac{u(z)}{h(z)}\right)^{2} h(z) h(x) m(d z) m(d x) .
\end{aligned}
$$

To this end, we first verify

$$
h(x) \approx \phi(\rho(x, y))^{\beta+1} / V(\rho(x, y)), \quad \rho(x, y)>0 .
$$

Indeed, letting $r=\rho(x, y)>0$ we first note that Lemma 9 yields $t \geq \phi(r)$ equivalent to $t V\left(\phi^{-1}(t)\right) \geq V(r) \phi(r)$, from whence

$$
\begin{aligned}
h(x) & \approx V(r)^{-1} \phi(r)^{-1} \int_{0}^{\phi(r)} t^{\beta+1} d t+\int_{\phi(r)}^{\infty} \frac{t^{\beta}}{V\left(\phi^{-1}(t)\right)} d t \\
& =(\beta+2)^{-1} \phi(r)^{\beta+1} / V(r)+I .
\end{aligned}
$$

To estimate $I$, we observe that the assumption $\phi \in \operatorname{WUSC}(\bar{\alpha}, \bar{c})$ implies $\phi^{-1} \in$ $\operatorname{WLSC}\left(1 / \bar{\alpha}, \bar{c}^{-1 / \bar{\alpha}}\right)[7$, Remark 4]. If $r>0$ and $t \geq \phi(r)$, then

$$
\begin{aligned}
\frac{V\left(\phi^{-1}(t)\right)}{V(r)} & \geq \frac{V\left(\phi^{-1}(t)\right)}{V\left(\phi^{-1}(\phi(r))\right)} \geq \underline{C}\left(\frac{\phi^{-1}(t)}{\phi^{-1}(\phi(r))}\right)^{\underline{A}} \\
& \geq \underline{C} \bar{c}^{-\underline{A} / \bar{\alpha}} \frac{t \underline{A} / \bar{\alpha}}{\phi(r) \underline{A} / \bar{\alpha}},
\end{aligned}
$$

hence,

$$
\frac{t^{\beta}}{V\left(\phi^{-1}(t)\right)} \leq \frac{\bar{c} \underline{A} / \bar{\alpha}}{\underline{C}} \frac{\phi(r) \underline{A} / \bar{\alpha} t^{\beta-\underline{A} / \bar{\alpha}}}{V(r)} .
$$

The claim (40) now follows because

$$
I \leq \frac{\bar{c}^{\underline{A} / \bar{\alpha}}}{\underline{C}} \frac{\phi(r)^{\underline{A} / \bar{\alpha}}}{V(r)} \int_{\phi(r)}^{\infty} t^{\beta-\underline{A} / \bar{\alpha}} d t=\frac{\bar{c} \underline{A} / \bar{\alpha}}{\underline{C}(\underline{A} / \bar{\alpha}-1-\beta)} \frac{\phi(r)^{\beta+1}}{V(r)} .
$$

The function

$$
k(x):=\int_{0}^{\infty} p_{t}(x, y)\left(t^{\beta}\right)^{\prime} d t, \quad x \in F,
$$

also satisfies

$$
k(x) \approx \phi(\rho(x, y))^{\beta} / V(\rho(x, y)), \quad x \in F .
$$

This follows by recalculating (40) for $\beta-1$. We get

$$
C_{1} \phi(\rho(x, y))^{-1} \leq q(x):=\frac{k(x)}{h(x)} \leq C_{2} \phi(\rho(x, y))^{-1},
$$

by choosing any $\beta \in(0, \underline{A} / \bar{\alpha}-1)$. The theorem follows from Eq. 16 . 
Remark 2 With the above notation, for each $0<\beta<\underline{A} / \bar{\alpha}-1$ there exists a constant $c$ such that for all $x, y \in F$ we have

$$
\int p_{t}(x, z) \phi(\rho(z, y))^{\beta+1} / V(\rho(z, y)) m(d z) \leq c \phi(\rho(x, y))^{\beta+1} / V(\rho(x, y))
$$

and

$$
\int \tilde{p}_{t}(x, z) \phi(\rho(z, y))^{\beta+1} / V(\rho(z, y)) m(d z) \leq c \phi(\rho(x, y))^{\beta+1} / V(\rho(x, y)),
$$

where $\tilde{p}$ is given by Eq. 10 with $q(x)=C_{1} \phi(\rho(x, y))^{-1}$ on $F$ and $C_{1}$ is the constant in the lower bound of the sharp estimate in Eq. 42 . This is a non-explosion result for $\tilde{p}$, and it is proved in the same way as Corollary 6.

Remark 3 Interestingly, the Chapman-Kolmogorov equations and Eq. 36 imply that $\phi$ in Theorem 10 satisfies a lower scaling, too. We leave the proof of this fact to the interested reader because it is not used in the sequel. An analogous situation occurs in [7, Theorem26].

In [11] a wide class of transition densities are constructed on locally compact separable metric measure spaces $(F, \rho, m)$ with metric $\rho$ and Radon measure $m$ of infinite mass and full support on $F$. Here are some of the assumptions of [11] (for details see [11, Theorem 1.2]). The functions $\phi, V:[0, \infty) \rightarrow(0, \infty)$ are increasing, $\phi(0)=V(0)=0, \phi(1)=1$, $\phi \in \operatorname{WLSC}(\underline{\alpha}, \underline{c}) \cap \operatorname{WUSC}(\bar{\alpha}, \bar{c}), V \in \operatorname{WLSC}(\underline{A}, \underline{C}) \cap \operatorname{WUSC}(\bar{A}, \bar{C})$, and

$$
\int_{0}^{r} \frac{s}{\phi(s)} d s \leq c \frac{r^{2}}{\phi(r)} \quad \text { for every } r>0 .
$$

A symmetric measurable function $J(x, y)$ satisfying

$$
J(x, y) \approx \frac{1}{V(\rho(x, y)) \phi(\rho(x, y))}, \quad x, y \in F, x \neq y,
$$

is considered in [11, Theorem 1.2] along with a symmetric pure-jump Markov process having $J$ as jump kernel and symmetric $p$ satisfying Eq. 36 as transition density. By Theorem 10, we obtain the following result.

Corollary 11 Under the assumptions of [11, Theorem 1.2], Eqs. 37 and 39 hold if $\underline{A}>\bar{\alpha}$.

We now specialize to $F=\mathbb{R}^{d}$ equipped with the Lebesgue measure. Let $v$ be an infinite isotropic unimodal Lévy measure on $\mathbb{R}^{d}$ i.e. $v(d x)=v(|x|) d x$, where $(0, \infty) \ni r \mapsto v(r)$ is nonincreasing, and

$$
v\left(\mathbb{R}^{d} \backslash\{0\}\right)=\infty \quad \text { and } \quad \int_{\mathbb{R}^{d} \backslash\{0\}}\left(|x|^{2} \wedge 1\right) v(d x)<\infty .
$$

Let

$$
\psi(\xi)=\int_{\mathbb{R}^{d}}(1-\cos \langle\xi, x\rangle) v(d x) .
$$

Because of rotational symmetry, $\psi$ depends only on $|\xi|$, and we can write $\psi(r)=\psi(\xi)$ for $r=|\xi|$. This $\psi$ is almost increasing [7], namely $\pi^{2} \psi(r) \geq \psi^{*}(r):=\sup \{\psi(p): 0 \leq p \leq$ $r$ \}. Let $0<\underline{\alpha} \leq \bar{\alpha}<2$. If $0 \not \equiv \psi \in \operatorname{WLSC}(\underline{\alpha}, \underline{c}) \cap \operatorname{WUSC}(\bar{\alpha}, \bar{c})$, then the following defines a convolution semigroup of functions,

$$
p_{t}(x)=(2 \pi)^{-d} \int_{\mathbb{R}^{d}} e^{i\langle\xi, x\rangle} e^{-t \psi(\xi)} d \xi, \quad t>0, x \in \mathbb{R}^{d},
$$


and the next two estimates hold [7, Theorem 21].

$$
\begin{aligned}
& p_{t}(x) \approx\left[\psi^{-}(1 / t)\right]^{d} \wedge \frac{t \psi(1 /|x|)}{|x|^{d}}, \quad t>0, x \in \mathbb{R}^{d}, \\
& v(|x|) \quad \approx \frac{\psi(1 /|x|)}{|x|^{d}}, \quad x \in \mathbb{R}^{d} .
\end{aligned}
$$

Here $\psi^{-}(u)=\inf \left\{s \geq 0: \psi^{*}(s) \geq u\right\}$, the left-continuous inverse of $\psi^{*}$. The corresponding Dirichlet form is

$$
\begin{aligned}
\mathcal{E}(u, u) & =(2 \pi)^{d} \int_{\mathbb{R}^{d}} \hat{u}(\xi) \overline{\hat{v}(\xi)} \psi(\xi) d \xi=\frac{1}{2} \int_{\mathbb{R}^{d}} \int_{\mathbb{R}^{d}}(u(x)-u(y))^{2} v(y-x) d y d x \\
& \approx \int_{\mathbb{R}^{d}} \int_{\mathbb{R}^{d}}(u(x)-u(y))^{2} \frac{\psi\left(|x-y|^{-1}\right)}{|y-x|^{d}} d y d x,
\end{aligned}
$$

cf. [19, Example 1.4.1] and the special case discussed in the proof of Proposition 5 above.

Corollary 12 If $d>\bar{\alpha}$, then is $c>0$ such that for all $u \in L^{2}\left(\mathbb{R}^{d}\right)$

$$
\mathcal{E}(u, u) \geq c \int_{\mathbb{R}^{d}} u(x)^{2} \psi(1 /|x|) d x .
$$

Proof Let $0<\beta<(d / \bar{\alpha})-1, h(x)=\int_{0}^{\infty} t^{\beta} p_{t}(x) d t$, and $k(x)=\int_{0}^{\infty}\left(t^{\beta}\right)^{\prime} p_{t}(x) d t$. Considering $\rho(x, y)=|y-x|, \phi(r)=1 / \psi(1 / r)$ and $V(r)=r^{d}$, by Eq. 37 we get Eq. 48 for all $u \in L^{2}\left(\mathbb{R}^{d}\right)$. To add some detail, we note that $\phi$ satisfies the same scalings as $\psi^{*}$ and $\phi^{-1}(t)=1 / \psi^{-}\left(t^{-1}\right)$. Thus Eq. 40 yields $h(x) \approx \psi\left(|x|^{-1}\right)^{-\beta-1}|x|^{-d}$ and $k(x) \approx \psi\left(|x|^{-1}\right)^{-\beta}|x|^{-d}$. In fact, we actually obtain Hardy equality. Indeed,

$$
\begin{aligned}
\liminf _{t \rightarrow 0} & \int_{\mathbb{R}^{d}} \int_{\mathbb{R}^{d}} \frac{p_{t}(x, y)}{2 t}\left[\frac{u(x)}{h(x)}-\frac{u(y)}{h(y)}\right]^{2} h(y) h(x) d y d x \\
& =\frac{1}{2} \int_{\mathbb{R}^{d}} \int_{\mathbb{R}^{d}}\left[\frac{u(x)}{h(x)}-\frac{u(y)}{h(y)}\right]^{2} h(y) h(x) v(|x-y|) d y d x,
\end{aligned}
$$

because if $t \rightarrow 0$, then $p_{t}(x, y) / t \leq c v(|x-y|)$ by Eqs. 46 and 47, and $p_{t}(x, y) / t \rightarrow$ $v(|x-y|$ ) (weak convergence of radially monotone functions implies convergence almost everywhere), and we can use the dominated convergence theorem or Fatou's lemma, as in the proof of Proposition 5. We thus have a strengthening of Eq. 48 for every $u \in L^{2}\left(\mathbb{R}^{d}\right)$ :

$$
\begin{aligned}
\mathcal{E}(u, u)= & \int_{\mathbb{R}^{d}} u(x)^{2} \frac{k(x)}{h(x)} d x \\
& +\frac{1}{2} \int_{\mathbb{R}^{d}} \int_{\mathbb{R}^{d}}\left[\frac{u(x)}{h(x)}-\frac{u(y)}{h(y)}\right]^{2} h(y) h(x) v(|x-y|) d y d x .
\end{aligned}
$$

For instance if we take $\psi(\xi)=|\xi| \sqrt{\log (1+|\xi|)}$, the Lévy-Kchintchine exponent of a subordinated Brownian motion [28], then we obtain

$$
\int_{\mathbb{R}^{d}}|\hat{u}(\xi)|^{2}|\xi| \sqrt{\log (1+|\xi|)} d \xi \geq c \int_{\mathbb{R}^{d}} \frac{u(x)^{2} \sqrt{\log \left(1+|x|^{-1}\right)}}{|x|} d x, \quad u \in L^{2}\left(\mathbb{R}^{d}\right) .
$$

Remark 4 We note that [14, Theorem 1, the "thin" case (T)] gives Eq. 48 for continuous functions $u$ of compact support in $\mathbb{R}^{d}$. Here we extend the result to all functions $u \in L^{2}\left(\mathbb{R}^{d}\right)$, 
as typical for our approach. We note in passing that [14, Theorems 1 and 5] offers a general framework for Hardy inequalities without the remainder terms and applications for quadratic forms on Euclidean spaces.

Here is an analogue of Remark 2.

Remark 5 Using the notation above, for every $0<\beta<(d-\bar{\alpha}) / \bar{\alpha}$, there exist constants $c_{1}$, $c_{2}$ such that

$$
\int p_{t}(y-x) \psi\left(|y|^{-1}\right)^{-\beta-1}|y|^{-d} d y \leq c_{1} \psi\left(|x|^{-1}\right)^{-\beta-1}|x|^{-d}, \quad x \in \mathbb{R}^{d},
$$

and

$$
\int \tilde{p}_{t}(x, y) \psi\left(|y|^{-1}\right)^{-\beta-1}|y|^{-d} d y \leq c_{1} \psi\left(|x|^{-1}\right)^{-\beta-1}|x|^{-d}, \quad x \in \mathbb{R}^{d},
$$

where $\tilde{p}$ is given by Eq. 10 with $q(x)=c_{2} \psi(1 /|x|)$ on $\mathbb{R}^{d}$. The result is proved as Remark 2. In particular we obtain non-explosion of Schrödinger perturbations of such unimodal transition densities with $q(x)=c_{2} \psi(1 /|x|)$. Naturally, the largest valid $c_{2}$ is of further interest.

\section{Weak Local Scaling on Euclidean Spaces}

In this section we restrict ourselves to the Euclidean space and apply Theorem 2 to a large class of symmetric jump processes satisfying two-sided heat kernel estimates given in [10] and [7]. Let $\phi: \mathbb{R}_{+} \rightarrow \mathbb{R}_{+}$be a strictly increasing continuous function such that $\phi(0)=0$, $\phi(1)=1$, and

$$
\underline{c}\left(\frac{R}{r}\right)^{\underline{\alpha}} \leq \frac{\phi(R)}{\phi(r)} \leq \bar{c}\left(\frac{R}{r}\right)^{\bar{\alpha}} \quad \text { for every } 0<r<R \leq 1 .
$$

Let $J$ be a symmetric measurable function on $\mathbb{R}^{d} \times \mathbb{R}^{d} \cap\{x \neq y\}$ and let $\kappa_{1}, \kappa_{2}$ be positive constants such that

$$
\frac{\kappa_{1}^{-1}}{|x-y|^{d} \phi(|x-y|)} \leq J(x, y) \leq \frac{\kappa_{1}}{|x-y|^{d} \phi(|x-y|)}, \quad|x-y| \leq 1,
$$

and

$$
\sup _{x \in \mathbb{R}^{d}} \int_{\left\{y \in \mathbb{R}^{d}:|x-y|>1\right\}} J(x, y) d y=: \kappa_{2}<\infty .
$$

We consider the quadratic form

$$
\mathcal{E}(u, u)=\frac{1}{2} \int_{\mathbb{R}^{d}} \int_{\mathbb{R}^{d}}[u(y)-u(x)]^{2} J(x, y) d y d x, \quad u \in L^{2}\left(\mathbb{R}^{d}, d x\right),
$$

with the Lebesgue measure $d x$ as the reference measure, for the symmetric pure-jump Markov processes on $\mathbb{R}^{d}$ constructed in [9] from the jump kernel $J(x, y)$.

Theorem 13 If $d \geq 3$, then

$$
\mathcal{E}(u, u) \geq c \int_{\mathbb{R}^{d}} u(x)^{2} \frac{d x}{\phi(|x|) \vee|x|^{2}}, \quad u \in L^{2}\left(\mathbb{R}^{d}\right) .
$$


Proof Let $\mathcal{Q}$ and $p_{t}(x, y)$ be the quadratic form and the transition density corresponding to the symmetric pure-jump Markov process in $\mathbb{R}^{d}$ with the jump kernel $J(x, y) \mathbf{1}_{\{|x-y| \leq 1\}}$ instead of $J(x, y)$, cf. [10, Theorem 1.4]. Thus,

$$
\mathcal{Q}(u, u)=\frac{1}{2} \int_{\mathbb{R}^{d} \times \mathbb{R}^{d}}(u(x)-u(y))^{2} J(x, y) \mathbf{1}_{\{|x-y| \leq 1\}} d x d y, \quad u \in C_{c}\left(\mathbb{R}^{d}\right) .
$$

We define $h$ as $h(x)=\int_{0}^{\infty} p_{t}(x, 0) t^{\beta} d t, x \in \mathbb{R}^{d}$ where $-1<\beta<d / 2-1$. We note that for every $T, M \geq 0$,

$$
\int_{T}^{\infty} t^{\beta-\frac{d}{2}} e^{-\frac{M r^{2}}{t}} d t=r^{2 \beta-d+2} \int_{0}^{\frac{r^{2}}{T}} u^{-2-\beta+\frac{d}{2}} e^{-M u} d u
$$

We shall use [10, Theorem 1.4]. We however note that the term $\log \frac{|x-y|}{t}$ in the statement of [10, Theorem 1.4] should be replaced by $1+\log _{+} \frac{|x-y|}{t}$, to include the case $c^{-1} t \leq$ $|x-y| \leq c t$ missed in the considerations in [10]. With this correction, our arguments are as follows. When $r=|x| \leq 1$, we have

$$
c_{0}^{-1}\left(\frac{1}{\phi^{-1}(t)^{d}} \wedge \frac{t}{r^{d} \phi(r)}\right) \leq p_{t}(x, 0) \leq c_{0}\left(\frac{1}{\phi^{-1}(t)^{d}} \wedge \frac{t}{r^{d} \phi(r)}\right), \quad t \in(0,1]
$$

and

$$
p_{t}(x, 0) \leq c_{0} t^{-d / 2} e^{-\bar{c}\left(\left(r\left(\log _{+}\left(\frac{r}{t}\right)+1\right)\right) \wedge \frac{r^{2}}{t}\right)} \leq c_{0} t^{-d / 2} e^{-\bar{c} \frac{r^{2}}{t}}, \quad t>1 .
$$

Thus, by Eq. 55, Lemma 9, Eqs. 56, 41, 51 and 54,

$$
\begin{aligned}
& c_{3} \frac{\phi(r)^{\beta+1}}{r^{d}} \leq c_{0}^{-1} \int_{0}^{\phi(r)} \frac{t^{\beta+1}}{r^{d} \phi(r)} d t \leq h(x) \\
\leq & c_{0} \int_{0}^{\phi(r)} \frac{t^{\beta+1}}{r^{d} \phi(r)} d t+c_{0} \int_{\phi(r)}^{1} \frac{t^{\beta}}{\left(\phi^{-1}(t)\right)^{d}} d t+c_{0} \int_{1}^{\infty} t^{\beta-\frac{d}{2}} e^{-\frac{\bar{c} r^{2}}{t}} d t \\
\leq & c_{4} \frac{\phi(r)^{\beta+1}}{r^{d}}+\frac{c_{5}}{r^{d-2-2 \beta}} \int_{0}^{\infty} u^{-2-\beta+d / 2} e^{-\bar{c} u} d u \\
\leq & c_{6}\left(\phi(r)^{1+\beta}+r^{2+2 \beta}\right) r^{-d} .
\end{aligned}
$$

If $r=|x|>1$, then by [10, Theorem 1.4], we have

$$
c_{0}^{-1} e^{-\underline{c} r\left(\log _{+}\left(\frac{r}{t}\right)+1\right)} \leq p_{t}(x, 0) \leq c_{0} e^{-\bar{c} r\left(\log _{+}\left(\frac{r}{t}\right)+1\right)}, \quad t \in(0,1],
$$

and for $t>1$ we have

$$
c_{0}^{-1} e^{-\underline{c}\left(\left(r\left(\log _{+}\left(\frac{r}{t}\right)+1\right)\right) \wedge \frac{r^{2}}{t}\right)} \leq p_{t}(x, 0) / t^{-d / 2} \leq c_{0} e^{-\bar{c}\left(\left(r\left(\log _{+}\left(\frac{r}{t}\right)+1\right)\right) \wedge \frac{r^{2}}{t}\right)} .
$$

In particular,

$$
p_{t}(x, 0) \geq c_{0}^{-1} t^{-d / 2} e^{-\underline{c}\left(\left(r\left(\log _{+}\left(\frac{r}{t}\right)+1\right)\right) \wedge \frac{r^{2}}{t}\right)} \geq c_{7} t^{-d / 2}, \quad t>r^{2} .
$$


Then Eqs. 57, 58, 59, 51 and 54 give

$$
\begin{aligned}
& \frac{c_{7}}{r^{d-2-2 \beta}} \int_{0}^{1} u^{-2-\beta+d / 2} d u=c_{7} \int_{r^{2}}^{\infty} t^{\beta-\frac{d}{2}} d t \leq h(x) \\
\leq & c_{8} \int_{0}^{r} t^{\beta} e^{-c_{9} r} d t+c_{8} \int_{r}^{\infty} t^{\beta-\frac{d}{2}} e^{-\frac{c_{10} r^{2}}{t}} d t \\
= & c_{8}(\beta+1)^{-1} r^{\beta+1} e^{-c_{9} r}+\frac{c_{8}}{r^{d-2-2 \beta}} \int_{0}^{r} u^{-2-\beta+d / 2} e^{-c_{10} u} d u \\
\leq & \frac{c_{11}}{r^{d-2-2 \beta}} .
\end{aligned}
$$

Thus,

$$
h(x) \approx\left(\phi(|x|) \vee|x|^{2}\right)^{\beta+1}|x|^{-d}, \quad x \in \mathbb{R}^{d} .
$$

In particular, if $0<\beta<d / 2-1$, and $k(x)=\int_{0}^{\infty} p_{t}(x, 0)\left(t^{\beta}\right)^{\prime} d t$, then

$$
k(x) \approx\left(\phi(|x|) \vee|x|^{2}\right)^{\beta}|x|^{-d}, \quad x \in \mathbb{R}^{d} .
$$

Therefore,

Theorem 2 yields

$$
q(x):=\frac{k(x)}{h(x)} \approx \frac{1}{\phi(|x|) \vee|x|^{2}} .
$$

$$
\mathcal{E}(u, u) \geq \mathcal{Q}(u, u) \geq c_{12} \int_{\mathbb{R}^{d}} u(x)^{2} \frac{d x}{\phi(|x|) \vee|x|^{2}}, \quad u \in L^{2}\left(\mathbb{R}^{d}\right) .
$$

Remark 6 As in Remark 5 we obtain non-explosion for Schrödinger perturbations by $q(x)=c /\left[\phi(|x|) \vee|x|^{2}\right]$.

Remark 7 The arguments and conclusions of Theorem 13 are valid for the unimodal Lévy processes, in particular for the subordinated Brownian motions, provided their LévyKhintchine exponent $\psi$ satisfies the assumptions of local scaling conditions at infinity with exponents strictly between 0 and $2<d$ made in [7, Theorem 21]:

$$
\int_{\mathbb{R}^{d}}|\hat{u}(\xi)|^{2} \psi(\xi) d \xi \geq c \int_{\mathbb{R}^{d}} u(x)^{2}\left[\psi\left(\frac{1}{|x|}\right) \wedge \frac{1}{|x|^{2}}\right] d x, \quad u \in L^{2}\left(\mathbb{R}^{d}\right) .
$$

Acknowledgments We thank William Beckner for comments on the Hardy equality (29). We thank Tomasz Byczkowski, Tomasz Grzywny, Tomasz Jakubowski, Kamil Kaleta, Agnieszka Kałamajska and Dominika Pilarczyk for comments, suggestions and encouragement. We also thank Rupert L. Frank and Georgios Psaradakis for remarks on the literature related to Section 4.

Open Access This article is distributed under the terms of the Creative Commons Attribution 4.0 International License (http://creativecommons.org/licenses/by/4.0/), which permits unrestricted use, distribution, and reproduction in any medium, provided you give appropriate credit to the original author(s) and the source, provide a link to the Creative Commons license, and indicate if changes were made.

\section{References}

1. Ancona, A.: On strong barriers and an inequality of Hardy for domains in $R^{n}$. J. London Math. Soc. (2) 34(2), 274-290 (1986) 
2. Andrews, G.E., Askey, R., Roy, R.: Special functions, volume 71 of Encyclopedia of Mathematics and its Applications. Cambridge University Press, Cambridge (1999)

3. Baras, P., Goldstein, J.A.: The heat equation with a singular potential. Trans. Amer. Math. Soc. 284(1), 121-139 (1984)

4. Beckner, W.: Pitt's inequality and the fractional Laplacian: sharp error estimates. Forum Math. 24(1), 177-209 (2012)

5. Bogdan, K., Byczkowski, T., Kulczycki, T., Ryznar, M., Song, R., Vondraċek, Z.: Potential analysis of stable processes and its extensions, volume 1980 of Lecture Notes in Mathematics. Springer, Berlin (2009). Edited by Piotr Graczyk and Andrzej Stos

6. Bogdan, K., Dyda, B.: The best constant in a fractional Hardy inequality. Math. Nachr. 284(5-6), 629638 (2011)

7. Bogdan, K., Grzywny, T., Ryznar, M.: Density and tails of unimodal convolution semigroups. J. Funct. Anal. 266(6), 3543-3571 (2014)

8. Bogdan, K., Hansen, W., Jakubowski, T.: Time-dependent Schrödinger perturbations of transition densities. Studia Math. 189(3), 235-254 (2008)

9. Chen, Z.-Q., Kim, P., Kumagai, T.: On heat kernel estimates and parabolic Harnack inequality for jump processes on metric measure spaces. Acta Math. Sin. (Engl. Ser.) 25(7), 1067-1086 (2009)

10. Chen, Z.-Q., Kim, P., Kumagai, T.: Global heat kernel estimates for symmetric jump processes. Trans. Amer. Math. Soc. 363(9), 5021-5055 (2011)

11. Chen, Z.-Q., Kumagai, T.: Heat kernel estimates for jump processes of mixed types on metric measure spaces. Probab. Theory Related Fields 140(1-2), 277-317 (2008)

12. Chen, Z.-Q., Song, R.: Hardy inequality for censored stable processes. Tohoku Math. J. (2) 55(3), 439450 (2003)

13. Dyda, B.: Fractional calculus for power functions and eigenvalues of the fractional Laplacian. Fract. Calc. Appl. Anal. 15(4), 536-555 (2012)

14. Dyda, B., Vähäkangas, A.V.: A framework for fractional Hardy inequalities. Ann. Acad. Sci. Fenn., Math. 39(2), 675-689 (2014)

15. Filippas, S., Tertikas, A.: Optimizing improved Hardy inequalities. J. Funct. Anal. 192(1), 186-233 (2002)

16. Fitzsimmons, P.J.: Hardy's inequality for Dirichlet forms. J. Math. Anal. Appl. 250(2), 548-560 (2000)

17. Frank, R.L., Lieb, E.H., Seiringer, R.: Hardy-Lieb-Thirring inequalities for fractional Schrödinger operators. J. Amer. Math. Soc. 21(4), 925-950 (2008)

18. Frank, R.L., Seiringer, R.: Non-linear ground state representations and sharp Hardy inequalities. J. Funct. Anal. 255(12), 3407-3430 (2008)

19. Fukushima, M., Oshima, Y., Takeda, M.: Dirichlet forms symmetric Markov processes, volume 19 of de Gruyter Studies in Mathematics. Walter de Gruyter \& Co. (2011)

20. Herbst, I.W.: Spectral theory of the operator $\left(p^{2}+m^{2}\right)^{1 / 2}-Z e^{2} / r$. Comm. Math. Phys. 53(3), 285-294 (1977)

21. Hille, E., Phillips, R.S.: Functional analysis and semi-groups. American Mathematical Society, Providence, R. I. (1974). Third printing of the revised edition of 1957, American Mathematical Society Colloquium Publications, Vol. XXXI

22. Jakubowski, T.: Fundamental solution of the fractional diffusion equation with a singular drift. preprint (2014)

23. Kałamajska, A., Pietruska-Pałuba, K.: On a variant of the Gagliardo-Nirenberg inequality deduced from the Hardy inequality. Bull. Pol. Acad. Sci. Math. 59(2), 133-149 (2011)

24. Kim, P., Song, R., Vondraciek, Z.: Global uniform boundary Harnack principle with explicit decay rate and its application. Stochastic Process. Appl. 124(1), 235-267 (2014)

25. Lax, P.D.: Functional analysis. Pure and Applied Mathematics (New York). Wiley, New York (2002)

26. Maz'ya, V.: Sobolev spaces with applications to elliptic partial differential equations, volume 342 of Grundlehren der Mathematischen Wissenschaften [Fundamental Principles of Mathematical Sciences]. Springer, Heidelberg (2011). augmented edition

27. Pilarczyk, D.: Self-similar asymptotics of solutions to heat equation with inverse square potential. J. Evol. Equ. 13(1), 69-87 (2013)

28. Schilling, R.L., Song, R., Z. Vondracek Bernstein functions volume 37 of de Gruyter Studies in Mathematics, second edition. Walter de Gruyter \& Co., Berlin (2012). Theory and applications

29. Skrzypczak, I.: Hardy-type inequalities derived from $p$-harmonic problems. Nonlinear Anal. 93, 30-50 (2013)

30. Vazquez, J.L., Zuazua, E.: The Hardy inequality and the asymptotic behaviour of the heat equation with an inverse-square potential. J. Funct. Anal. 173(1), 103-153 (2000)

31. Zähle, M.: Potential spaces and traces of Lévy processes on $h$-sets. Izv. Nats. Akad. Nauk Armenii Mat. 44(2), 67-100 (2009) 